\title{
Role of Flagella in Adhesion of Escherichia coli to Abiotic Surfaces
}

\section{Citation}

Friedlander, Ronn S., Nicolas Vogel, and Joanna Aizenberg. .2015. On the role of flagella in adhesion of Escherichia coli to abiotic surfaces. Langmuir 31, no. 22: 6137-6144. D0I: 10.1021/ acs.langmuir.5b00815

\section{Published Version}

doi:10.1021/acs.langmuir.5b00815

\section{Permanent link}

http://nrs.harvard.edu/urn-3:HUL.InstRepos:33204050

\section{Terms of Use}

This article was downloaded from Harvard University's DASH repository, and is made available under the terms and conditions applicable to Open Access Policy Articles, as set forth at http:// nrs.harvard.edu/urn-3:HUL.InstRepos:dash.current.terms-of-use\#OAP

\section{Share Your Story}

The Harvard community has made this article openly available.

Please share how this access benefits you. Submit a story.

\section{Accessibility}


On the role of flagella in adhesion of Escherichia coli to abiotic surfaces

\author{
Ronn S. Friedlander ${ }^{1,2}$, Nicolas $\operatorname{Vogel}^{3-5}$, Joanna Aizenberg ${ }^{*}, 2,3,6$
}

1. Harvard-MIT division of Health Sciences and Technology, Cambridge, MA 02139 (USA)

2. Wyss Institute for Biologically Inspired Engineering, Harvard University, 29 Oxford Street, Cambridge MA 02138 (USA)

3. School of Engineering and Applied Sciences, Harvard University, Cambridge, MA 02138 (USA)

4. Institute of Particle Technology, Department of Chemical and Biological Engineering, Friedrich-Alexander University Erlangen-Nürnberg (Germany)

5. Cluster of Excellence - Engineering of Advanced Materials, Friedrich Alexander-University ErlangenNürnberg (Germany)

6. Department of Chemistry and Chemical Biology, Harvard University, Cambridge, MA 02138 (USA)

Abstract: Understanding the interfacial activity of bacteria is of critical importance due to the huge economic and public health implications associated with surface fouling and biofilm formation. The complexity of the process and difficulties of predicting microbial adhesion to novel materials demand study of the properties of specific bacterial surface features and their potential contribution to surface attachment. Here, we examine flagella, cell appendages primarily studied for their cell motility function, to elucidate their role in surface adhesion of Escherichia coli-a model organism and potential pathogen. We use self-assembled monolayers (SAMs) of thiol-bearing molecules on gold films to generate surfaces of varying hydrophobicity, and measure adhesion of purified flagella using quartz crystal microbalance. We show that flagella adhere more extensively and bind more tightly to hydrophobic SAMs than to hydrophilic ones, and propose a two-step vs a single-step adhesion mechanism that accounts for the observed dissipation and frequency changes for the two types of surfaces, respectively. Subsequently, study of the adhesion of wild-type and flagella knock-out cells confirms that flagella improve adhesion to hydrophobic substrates, whereas cells lacking flagella do not show preferred affinity to hydrophobic substrates. Together, these properties bring about an interesting ability of cells with flagella to stabilize emulsions of aqueous culture and dodecane, not observed for cells lacking flagella. This work contributes to our overall understanding of nonspecific bacterial adhesion and suggests that flagella, beyond motility, may play an important role in surface adhesion.

\title{
Introduction:
}


Bacterial surface adhesion is a ubiquitous phenomenon in the natural world, and as the first step in biofilm formation, it enables microbes to survive changing environments, chemical and physical assaults and depletion of resources ${ }^{1,2}$. Due to the survival advantages imparted by the biofilm lifestyle, pristine, unoccupied surfaces provide high-value real estate to bacteria, particularly when conditions favor a survival mode, rather than a proliferative one ${ }^{3}$. Unfortunately, numerous materials applications require the maintenance of pristine surfaces in bacteria-rich environments. These include remote optical sensors, biomedical materials and marine vessels, to name a few ${ }^{3-6}$. Often, these surfaces become exposed to bacteria during use and are ultimately fouled by adhering microbes. Once these microbes progress to mature biofilms, they become difficult or impossible to remove ${ }^{7}$. The prevention of bacterial adhesion to surfaces has thus been an important, interdisciplinary field of research. Different strategies have evolved, focusing on surface chemistry, topographic patterns or cytotoxicity ${ }^{8,9}$. These have met with varying levels of success but to this point, the challenge remains.

The design of superhydrophobic surfaces appears to be a promising starting point to nonspecifically prevent bacteria from adhering. By combining hydrophobic surface chemistry with micro- or nano-scale topography, such surfaces become extremely repellent since water is prevented from coming into contact with the complete topography and resides on a metastable composite air/solid interface atop the structures ${ }^{10-13}$. As a result, the contact area between liquid and surface is minimized, leading to ease of removal of the droplet. Unfortunately, we have found that the combination of hydrophobicity and structure does not work as expected in repelling water-borne bacteria ${ }^{14}$. On the contrary, bacteria can adsorb, cover the surface and degrade the metastable air/solid interfaces at the top of the microstructures, leading to the loss of superhydrophobicity and an increase in available surface area. Consequently, such surfaces actually increase bacterial adhesion compared to flat ones.

Since bacterial adhesion is a complex process that depends on the specific environments, materials and species involved, potential rearrangements and changes of observed effects in the course of the adhesion process as well as temporal variations in cell surface composition, it is difficult to generalize performance of antifouling materials. ${ }^{15-19}$ Therefore, a detailed understanding of the mechanisms of adhesion, cell surface features and physical properties is of 
paramount importance in order to advance our ability to design anti-adhesive materials and predict performance.

In the process of adhesion, bacteria can rely on either specific adhesive organelles or proteins (e.g. pili, lipopolysaccharide, non-fimbrial adhesin-1), or nonspecific interactions with surfaces such as van der Waals, electrostatic, and hydrophobic interactions ${ }^{20-22}$. Escherichia coli has been well studied as a laboratory model organism in general, and in adhesion and biofilm formation in particular. It has been shown that specific flagellar isoforms can enable attachment of certain E. coli strains to mammalian antigens. For example the flagellar filament of $E$. coli Nissle 1917 adheres to porcine mucin $2^{23}$, and a secreted protein, EtpA, can mediate adhesion between the tips of enterotoxigenic $E$. coli flagellar filaments and host cell surface receptors ${ }^{24}$.

Characterizations of other specific adhesive interactions have exposed new potential targets for anti-adhesive strategies. For example, some studies of E. coli adhesion as it relates to virulence have focused on type I pili ${ }^{20,25-27}$, which can bind to mannose on eukaryotic cell surfaces. Pilus-mediated adhesion has been specifically targeted by the development of pilicides, which block pili biogenesis ${ }^{28}$. E. coli can also secrete extracellular polymeric substances, such as curli (an amyloid fiber) and colanic acid or cellulose (polysaccharides). These substances can act as virulence factors by enabling cells to improve adhesion to tissues and assemble biofilms ${ }^{29-32}$. Similar in strategy to pilicides, curlicides have also been developed to inhibit curli biogenesis ${ }^{33}$. Such strategies target virulence rather than microbial death, which results in reduced selection pressure for resistance relative to antibiotics (a major and growing concern in public health). While promising, pilicides and curlicides target specific cell-host interactions but do not address nonspecific bacterial adhesion to inert biomaterials, which can act as portals and reservoirs for infection.

Some surface organelles and molecules have been examined for their nonspecific adhesive properties. For example, a study of type I fimbriae indicated that these organelles strengthened adhesion to hydrophobic surfaces, but reduced adhesion to hydrophilic surfaces ${ }^{34}$. Another study focusing on lipopolysaccharide (LPS) showed that the length and charge of LPS molecules on the surface of $E$. coli K12 strains did not directly correlate with adhesion to quartz particles ${ }^{35}$. We recently showed that bacterial flagella can promote adhesion to structured abiotic surfaces by (i) providing vibrational energy near the topographic features that induces surface 
wetting in superhydrophobic coatings and (ii) mending the substrate with a flagella "carpet", enabling bacteria to overcome the geometric constraints size imposed by the size and shape of the cell body relative to surface features ${ }^{14}$. However, the role of flagella in adhesion to biomaterials and abiotic surfaces more generally has not been well described.

In the present study, we aim to shed light on the role of flagella for E. coli adhesion via non-specific interactions by quantifying attachment of isolated flagella to surfaces with different hydrophobicities. We show that purified flagella adhere to hydrophobic surfaces, and that cells possessing flagella demonstrate increased adhesion to hydrophobic surfaces compared to their non-flagellated counterparts. We also quantify adhesion of whole cells to hydrophobic and hydrophilic surfaces, comparing strains with and without surface appendages. We show that flagella increase cell surface activity at oil/water interfaces. Surprisingly, we also see that flagella adhere poorly to hydrophilic surfaces and reduce adhesion of the cell to these interfaces, contrasting with the widely held view that flagella categorically improve adhesion and biofilm formation of $E$. coli on immersed surfaces ${ }^{20,36-38}$. This work highlights and elucidates the important role that flagella may play in surface adhesion, beyond their role in cell motility.

\section{Experimental:}

Strains and culture conditions. All strains used in this study are indicated with their sources in Table 1. For all adhesion assays, overnight cultures of E. coli were grown in Luria-Bertani (LB) broth shaking at $220 \mathrm{RPM}$ at $37^{\circ} \mathrm{C}$. Overnight cultures were diluted 1:100 in M63 salts plus $0.5 \%(\mathrm{w} / \mathrm{v})$ casamino acids and $0.2 \%(\mathrm{w} / \mathrm{v})$ glucose $\left(\mathrm{M}^{+} 3^{+}\right)$. When needed, kanamycin was used at $75 \mu \mathrm{g} / \mathrm{ml}$. Gold-coated glass coupons with thiol SAMs were placed at the bottoms of 4-well rectangular plates, so that each well contained one hydrophobic and one hydrophilic coupon in a randomized position. The overnight cultures diluted in $\mathrm{M}_{6} 3^{+}$were used to cover these coupons and the plates were incubated at $37^{\circ} \mathrm{C}$ under static conditions for the times indicated in each experiment. At the endpoints, coupons were rinsed with phosphate-buffered saline (PBS) so as to remove loosely adherent cells. In order to avoid dewetting of the surfaces, PBS was flooded into the wells and allowed to overflow and dilute the medium. To keep shear forces consistent between experiments, wells were rinsed using a peristaltic pump at a flow rate of 100 
$\mathrm{mL} / \mathrm{min} /$ well. Each well was rinsed with a total of $600 \mathrm{~mL}$ of PBS. After rinsing, half of the liquid volume in each well was removed and $2 \mathrm{x}$ fixative was added (4\% glutaraldehyde, $5 \%$ paraformaldehyde in $0.16 \mathrm{M}$ Sorenson's phosphate buffer), to obtain $1 \mathrm{x}$ fixative in the well. This protocol enables the samples to remain immersed in liquid at all times and reliably avoids dewetting of hydrophobic samples. Fixative was incubated for $15 \mathrm{~min}$ and then rinsed with 20 volumes of PBS with a peristaltic pump at $100 \mathrm{~mL} / \mathrm{min} /$ well, again avoiding dewetting.

Growth Curves. To ensure the various strains of E. coli grew at comparable rates under the same conditions, we measured growth curves for each. Stationary phase cultures (from overnight growth) were inoculated at an effective optical density measured at $600 \mathrm{~nm}\left(\mathrm{OD}_{600}\right)$ of 0.05 in $150 \mu \mathrm{L}$ of medium of interest per well of a honeycomb well plate (Growth Curves USA). Plates were incubated in a Bioscreen $\mathrm{C}$ (Growth Curves USA) plate reader and set to shake continuously at high amplitude at $37^{\circ} \mathrm{C}$ for $18 \mathrm{~h} . \mathrm{OD}_{600}$ readings were taken every $10 \mathrm{~min}$.

Purification of flagella. Methods for isolating flagella were adapted from Crawford et al., 2010 and Erdem et al., $2007^{39,40}$. Cells were grown to exponential phase in $2.4 \mathrm{~L}$ of M63 ${ }^{+}$medium $\left(\mathrm{OD}_{600} \approx 0.6\right)$, at which point cells were harvested and centrifuged at $8000 \mathrm{rcf}$ for $15 \mathrm{~min}$. A small sample of cells was also examined under phase contrast microscope to check for motility. Supernatant was discarded and cells were resuspended in $250 \mathrm{~mL}$ PBS. The centrifugation step was repeated and cells were then resuspended in approximately $50 \mathrm{~mL}$ PBS. This suspension was blended for $60 \mathrm{~s}$ in a Waring commercial blender to shear off flagella. Cells were checked for loss of motility under microscope. Cell/flagella suspension was then centrifuged at $9000 \mathrm{rcf}$ for $20 \mathrm{~min}$ and then the supernatant was removed and spun at $12000 \mathrm{rcf}$ for $30 \mathrm{~min}$. Supernatant was removed and ammonium sulfate was slowly added to $45 \%$ saturation. This solution was incubated at $4{ }^{\circ} \mathrm{C}$ overnight. The next day, the solution was centrifuged at $12000 \mathrm{rcf}$ for $1 \mathrm{~h}$. The pellet was resuspended in a small volume $(\sim 2-6 \mathrm{~mL})$ of PBS while rocking at $4{ }^{\circ} \mathrm{C}$ for $8 \mathrm{~h}$. This solution was dialyzed against PBS using several buffer changes over 4 days. Concentration was measured using Coomassie Brilliant Blue $\mathrm{G}$ and standard curves obtained from BSA. If sample needed additional concentration, we used a Macrosep centrifugal device (Pall Corp.) with a 10 $\mathrm{kDa}$ molecular weight cutoff, following manufacturer's instructions. Purity was verified using SDS-PAGE with SimplyBlue Safe stain (Life Technologies). Morphology of purified flagellar filaments was examined using transmission electron microscopy. 
Gold-thiol SAMs. We prepared stock solutions of 11-mercapto-1-undecanol and dodecanethiol with a concentration of $100 \mathrm{mmol} / \mathrm{L}$ in ethanol. For the individual samples, we used these stock solutions to mix the two thiols in molar ratios as specified in the text and Figure 1 with a total concentration of $2 \mathrm{mmol} / \mathrm{L}$ in ethanol. We coated microscope slides with $2 \mathrm{~nm}$ titanium and 30 nm of gold using a Cressington 308 evaporator (Ted Pella, USA). The titanium served as an adhesion promoter to prevent delamination of the gold film. For bacterial adhesion experiments, we cut the slides into $1 \times 1 \mathrm{~cm}^{2}$ coupons and immersed them for $18 \mathrm{~h}$ in the thiol solutions. For the QCM-D experiments, we used commercially available gold-coated QCM-D crystals (Biolin Scientific, Inc.). To remove excess thiols, we rinsed the samples twice in ethanol and dried them under a stream of nitrogen gas immediately before using them in bacterial adhesion experiments.

Transmission electron microscopy (TEM). Samples of purified flagella were loaded onto a carbon-coated TEM grid. Grids were negatively stained with 1\% phosphotungstic acid for $10 \mathrm{~s}$, and then rinsed twice with deionized water. Grids were allowed to dry prior to TEM imaging. TEM images were acquired using a JEOL 2100 microscope with an operating voltage of $200 \mathrm{kV}$. High resolution images were taken using a Gatan Osiris digital camera.

Contact angle measurements. Static water contact angles were measured using a goniometer (CAM 101, KSV Instruments) at ambient condition with a $3 \mu 1$ droplet of water and evaluated with the provided software. All measurements were repeated at least five times on different areas of the substrates and averaged.

QCM-D. Gold-coated quartz crystals were obtained from Biolin Scientific, Inc. Prior to functionalization, crystals were cleaned by $10 \mathrm{~min}$ UV ozone exposure, followed by a $10 \mathrm{~min}$ bath in 5:1:1 $\mathrm{H}_{2} \mathrm{O}: \mathrm{H}_{2} \mathrm{O}_{2}: \mathrm{NH}_{4} \mathrm{OH}$, rinsing withe deionoized water (milliQ grade) and then another 10 min UV ozone exposure. Thiol SAMs were then assembled on the surfaces to obtain surfaces with a range of hydrophobicity following the protocol described above. Crystals were rinsed in $100 \%$ ethanol and dried under a stream of $\mathrm{N}_{2}$. Crystals were loaded into flow cell chambers of a Q-Sense E4 QCM-D and chambers were flushed with PBS. Purified flagella were diluted in PBS to a concentration of $5 \mu \mathrm{g} / \mathrm{mL}$. During measurements, flagella solution was flowed through the chambers at $10 \mu \mathrm{L} / \mathrm{min}$ until frequency change saturated and stabilized. Chambers were then flushed with PBS. 
Construction of mutants. To generate deletion mutants, genes of interest were replaced with kanamycin resistance cassettes, as described in Friedlander et al., $2013^{14}$. Briefly, mutations were transferred from Keio collection knockouts to the ZK2686 background strain via P1vir phage transduction ${ }^{41}$. Transductants were confirmed by polymerase chain reaction using primers internal to the kanamycin gene and upstream of the disrupted gene. For double deletion mutants, second deletions were introduced via another round of P1 vir phage transduction as described above, with a different selection marker.

Quantification of cell adhesion. After cell growth on SAMs for $4 \mathrm{~h}$, cells were rinsed and fixed as described in "Strains and culture conditions". Fixed cells in PBS were imaged on an inverted microscope with a water immersion lens. For each sample, 4-7 images were taken and cells were counted on each image and normalized to the surface area of the images. Each condition was repeated at least 4 separate times.

Two-phase cell mixtures. E. coli were grown overnight in LB, shaking at $220 \mathrm{RPM}$ at $37{ }^{\circ} \mathrm{C}$.

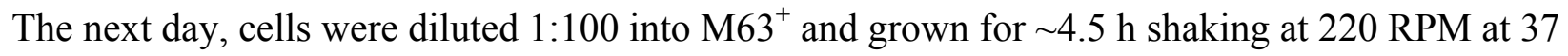
${ }^{\circ} \mathrm{C}$ to obtain an $\mathrm{OD}_{600}$ of 1.0 . Wild-type cells were checked for motility by phase contrast microscopy. $2 \mathrm{~mL}$ of this exponential phase cell suspension was placed in a glass culture tube. To this, $400 \mu \mathrm{L}$ of dodecane was added and tubes were vortexed on high speed for $1 \mathrm{~min}$. Tubes were allowed to rest for $10 \mathrm{~min}$ and were then photographed to image extent of phase reseparation. For microscopic imaging, emulsions were spotted onto glass slides and imaged using a phase contrast microscope. Certain samples of cells were stained with Syto9 live cell stain prior to emulsifying. These emulsions were imaged using fluorescence microscopy.

\section{Results and Discussion:}

Bacterial flagella are complex organelles that improve cell motility, enable chemotaxis, contribute to biofilm structure ${ }^{42}$, and aid in mucin adhesion for a number of species ${ }^{23,40,43-46}$. Flagella are the longest extensions from the cell surface and as such, they mediate many important interactions, both advantageous for the bacterium (e.g. epithelial cell adhesion) and deleterious (e.g. stimulation of innate immunity of higher organisms through Toll-like receptor-

5) ${ }^{47,48}$. By characterizing and quantifying the ability of flagella to adhere to abiotic surfaces and 
relating this to overall cell adhesive ability, we sought to better understand the diverse functionality of flagella, particularly by elucidating their functions beyond motility.

In order to isolate the effects of flagellar adhesion from other adhesive phenomena related to the cell body or other surface appendages, we purified flagellar filaments from $E$. coli ZK2686 (Figure 1A,B) and used them for quartz crystal microbalance with dissipation (QCM-D) measurements. QCM-D uses changes in resonance frequency of a quartz crystal and is extremely sensitive to changes in adsorbed mass $\left(\sim \mathrm{ng} / \mathrm{cm}^{2}\right)$, allowing us to precisely determine extent of flagella binding to the crystal surface. In order to control surface hydrophobicity, we used gold-coated crystals and generated self-assembled monolayers (SAMs) with thiol-bearing molecules ${ }^{49,50}$. We chose 11-mercapto-1-undecanol as a hydrophilic, uncharged functionality (water contact angle $28^{\circ}$ ) and dodecanethiol as a hydrophobic functionality (water contact angle $112^{\circ}$ ) and mixed them in different ratios to achieve intermediate hydrophilicities (Figure 1C-E). This blend of thiols enabled us to vary hydrophobicity without significantly altering other aspects of the surface, such as chemical structure or charge. It has been shown that such mixed monolayers do not show phase separation and lead to a homogeneous surface coating ${ }^{51-53}$. Since the size of the two molecules is similar, no topographic effects (i.e. shielding of one function by the other, larger molecule) are expected. It has previously been shown that dodecanethiol is preferentially incorporated from solution into mixed SAMs with 11-mercapto-1-undecanol ${ }^{53}$. This is consistent with our observation of water contact angles of $>90^{\circ}$ until the mole fraction of 11-mercapto-1-undecanol exceeds approximately 0.5 .

Quartz crystals with SAMs representing the full range of available hydrophobicities were chosen for flagellar adhesion experiments. Crystals were placed in flow chambers and solutions of $5 \mu \mathrm{g} / \mathrm{mL}$ of purified flagella in phosphate buffered saline (PBS) were flowed over the crystals at $10 \mu \mathrm{L} / \mathrm{min}$. Frequency changes were monitored during flow until these values saturated (Figure 1F). After several experiments for each condition, the final change in frequency, $\Delta f$, was averaged and plotted against the water contact angle of the SAM-modified surface as a measure of its hydrophobicity. We observed a general increase in adsorbed mass as hydrophobicity of the surfaces increased (Figure $1 \mathrm{G}$ ). The mean frequency shift saturated at approximately $-40 \mathrm{~Hz}$, which was achieved for surfaces with contact angles of $80^{\circ}$ and greater (SAMs assembled from a 
solution of 0.4 or greater mole fraction of dodecanethiol blended with 11-mercapto-1undecanol). The most hydrophilic surfaces allowed only very low flagella adhesion.

The viscoelasticity of the adsorbed material in our QCM measurements causes damping of the crystal vibrations, which is measured with QCM-D. In general, a softer or less tightly bound material will show stronger dissipation compared to a stiff film ${ }^{54}$. In the case of our filamentous flagella, more tightly bound filaments should result in less dissipation than loosely bound ones, which would increase drag and energy dissipation. Examining dissipation versus frequency (Figure 2A), we observe that the relationship is nearly linear for hydrophilic surfaces, but for hydrophobic surfaces, the dissipation-frequency slope decreases in magnitude at higher frequency changes. This indicates that the flagella become more tightly bound to hydrophobic interfaces. Normalizing for the total adsorbed mass (measured as $\Delta f$ ), we can also examine dissipation caused by bound flagella over the course of an experiment (Figure 2B). These plots indicate that on hydrophilic surfaces energy dissipation is highest at the onset of adhesion, and decreases exponentially. The more hydrophobic surfaces have distinct maxima of dissipation, followed by a decrease to a steady state. This corresponds to a more complex adhesive process with a changing dissipation profile.

We used the time-derivative of the frequency change to examine kinetics of adsorption throughout the QCM experiments (Figure 2C). We observe a monotonic decay of adsorption rate of flagella to hydrophilic surfaces over time, indicating surface saturation. For more hydrophobic crystals there is a complex behavior consisting of an increase to a plateau value, followed by a peak and decay of adsorption rate. This indicates a two-step adhesion profile with potential surface rearrangements, as observed previously. ${ }^{18,19}$ The maximal rate of adsorption tended to increase with hydrophobicity, but the overall maximum rate observed was to surfaces with contact angles of $\sim 80^{\circ}$, which decreased with higher contact angles. The peaks in adhesion rates observed for more hydrophobic surfaces can be interpreted in the context of frequency and dissipation data. As normalized dissipation begins to drop, there is a brief and coincident increase in rate of frequency change to a peak value (see dotted lines in Figure 2), which may indicate that the surface configuration of flagella adhering to hydrophobic surfaces is changing. Overall energy dissipation does not generally decrease—only normalized dissipation decreases, indicating a decrease in average energy dissipation per attached flagellum. 
Mechanistically, we interpret the difference between attachment to hydrophilic and hydrophobic surfaces in terms of a single versus multistep adhesion process, respectively. On hydrophilic surfaces, flagella adhere and are loosely bound to the surface and the tethered filaments continue to move in Brownian motion and obstruct additional attachment after adsorption of an initial layer, comparable to a steric repulsion process observed on polymers tethered to the surfaces of colloids ${ }^{55,56}$. This type of adsorption process would translate into a monotonic decay in adsorption rate and a linear relation between dissipation and frequency change as we observe in the QCM-D experiments. On hydrophobic surfaces however, we speculate that flagella are first attaching loosely; as in the case of hydrophilic surfaces. Over time, they seem to change their conformation and may "zip" onto the surface, becoming more tightly bound, most likely by increasing van-der-Waals attractions between flagella proteins and surface and progressive removal of water. ${ }^{18,19}$ This tighter binding would translate into a drop in normalized dissipation - as we observe in QCM-D. As the flagella "zip" onto the surface, the volume above the surface they shield by Brownian motion is decreased, allowing additional flagella to gain access and adsorb to the surface. Consistent with this interpretation, the decrease in dissipation we observe in QCM-D is followed by an increase in adhesion rate. We and others have previously observed flagellar entanglement in biofilms as well as highly flagellated strains at the substrate interface ${ }^{14,42}$, which is in agreement with such an interaction.

Regardless of the exact mechanism of the flagella-surface interaction, we have shown that flagella can adhere directly to surfaces of a hydrophobic nature and do not adhere as readily to hydrophilic surfaces. We emphasize that the behavior discussed here refers to mixed monolayers of non-charged species and should not be generalized to different types of surface chemistries that may include charged moieties or complex chemical functionalities, which may influence or alter the adhesion process. Nevertheless, our results suggest a possible dual role that flagella may play in surface adhesion. To verify this hypothesis and isolate the role of flagella in whole cell adhesion, we compared surface attachment of wild-type cells with that of mutants lacking flagellar filaments $(\Delta f l i C)$, lacking type I pili $(\Delta f i m H)$, and lacking both flagella and pili $(\Delta f l i C \Delta f i m H)$. All strains were derived from E. coli ZK2686 (Table 1), and growth curves were shown to be comparable (Figure 3A). To simplify, we constrained our experiments to the assessment of cell adhesion on purely hydrophilic and hydrophobic substrates, prepared by SAMs of dodecanethiol (hydrophobic) and 11-mercapto-1-undecanol (hydrophilic) on gold- 
coated microscope slides. Substrates were submerged in $\mathrm{M}^{+} 3^{+}$medium and sample wells were each inoculated with an E. coli strain. After 4 h, samples were washed with PBS using a peristaltic pump to control shear, while avoiding sample dewetting, as passage of an air-liquid interface can result in shear forces 100-1000 times greater than fluid flow alone ${ }^{21,57,58}$, which would distort the measured quantity of attached cells. Samples were then fixed and imaged with a light microscope for cell counts (Figure 3B-D).

In agreement with our previous study ${ }^{14}$, wild-type cell counts were highest on hydrophobic surfaces, and nearly 3-fold higher than cell counts on hydrophilic surfaces. Interestingly, loss of type I fimbriae did not negatively affect the ability of cells to adhere to hydrophobic surfaces, and possibly improved adhesion to hydrophilic surfaces. Loss of flagella strongly reduced the capacity of wild-type E. coli to adhere to hydrophobic surfaces, but significantly improved cell adhesion to hydrophilic surfaces, indicating some inhibitory effect of flagella in cell attachment to hydrophilic surfaces. For $\Delta f l i C$ cells, adhesion to hydrophilic and hydrophobic surfaces was nearly equal. Surprisingly, $\Delta$ fim $H \Delta f l i C$ cells were the most adherent strain for hydrophilic substrates. They also demonstrated similar levels of adhesion to hydrophilic and hydrophobic surfaces. We interpret these results to mean that the cell surface properties enable adhesion to hydrophilic surfaces, but the presence of flagella (which do not adhere well to hydrophilic surfaces) hinder this interaction, possibly due to their large size, movement and/or peritrichous arrangement. Nonetheless, wild-type cells adhere to hydrophobic surfaces better than $\Delta f l i C$ mutants, demonstrating a quantifiable advantage for possessing flagella in scenarios where hydrophobic surfaces are present.

Our finding that flagella displayed increased adhesion to hydrophobic surfaces and cells lacking flagella or fimbriae improved adhesion to hydrophilic surfaces raises an interesting possibility that the presence of flagella imparts an overall amphiphilicity to the cells. To test this hypothesis, we adapted a traditional method called "microbial adhesion to hydrocarbons" 59 . We mixed exponential phase cultures with dodecane to create a two-phase system. We emulsified the two phases by vortexing and investigated the stability of the emulsion over time (Figure 4). Indeed, we observed a stable emulsion over $2 \mathrm{~h}$, created in the presence of wild-type cells. Cells lacking flagella $(\triangle f l i C)$ did not create stable emulsions, and complete phase separation was observed within 5-10 min. We further examined the wild-type emulsions using optical 
microscopy (Figure 4C,D). Wild-type cells mixed with dodecane were enriched around the perimeters of large droplets, and formed clusters of cells that surrounded and stabilized smaller droplets. This result indicates that the flagella become embedded in the dodecane phase, and the more hydrophilic cell bodies remain associated with the aqueous phase, creating a living analog of a surfactant.

Based on our QCM-D findings as well as our results that cells lacking flagella adhere better to hydrophilic surfaces than wild-type cells, we can think of these cells as having hydrophobic flagellar filaments that interact with the hydrocarbon phase, and hydrophilic cell bodies, that preferentially interact with the aqueous phase. Essentially, the entire cell can act as a surfactant when it expresses flagella. In general, this property may improve surface activity of the cells, which is favorable for attachment and biofilm formation.

\section{Conclusion:}

This work allows us to better correlate live-cell adhesion experiments with cell surface properties and emphasizes the importance of characterizing and correlating both materials and cell properties during adhesion studies. The finding that flagella decrease adhesion to hydrophilic model surfaces belies the concept that flagella generally improve adhesion. The interplay between surface properties and bacterial physiology is complex and often unpredictable. Native environments provide variation in surface chemistry, topography, shear forces, pressures, osmolarity, ionic strength and nutrients. These properties are all likely to affect adhesion and thus, there is no adequate substitute for testing materials in their intended environments. Nonetheless, a deeper understanding of the material properties that relate to adhesion can be reached by abstracting material properties into relevant experimental systems, such as the hydrophobic-hydrophilic spectrum described here.

As large, extracellular appendages, flagella require significant cell resources and must therefore provide a substantial selective advantage to the cell, particularly for cells possessing multiple flagella, such as E. coli. We have shown that flagella have a complex and significant role in surface adhesion, which stands in addition to their function in cell motility. This may increase the return on investment cells receive from their flagella. With this in mind, we can 
think of flagella as multifunctional appendages that increase the cell's overall Darwinian fitness in multiple ways. Our description of flagella adhesive properties improves understanding of this complex and well-studied organelle and contributes insight to the lifestyle of $E$. coli.

\section{Figures:}
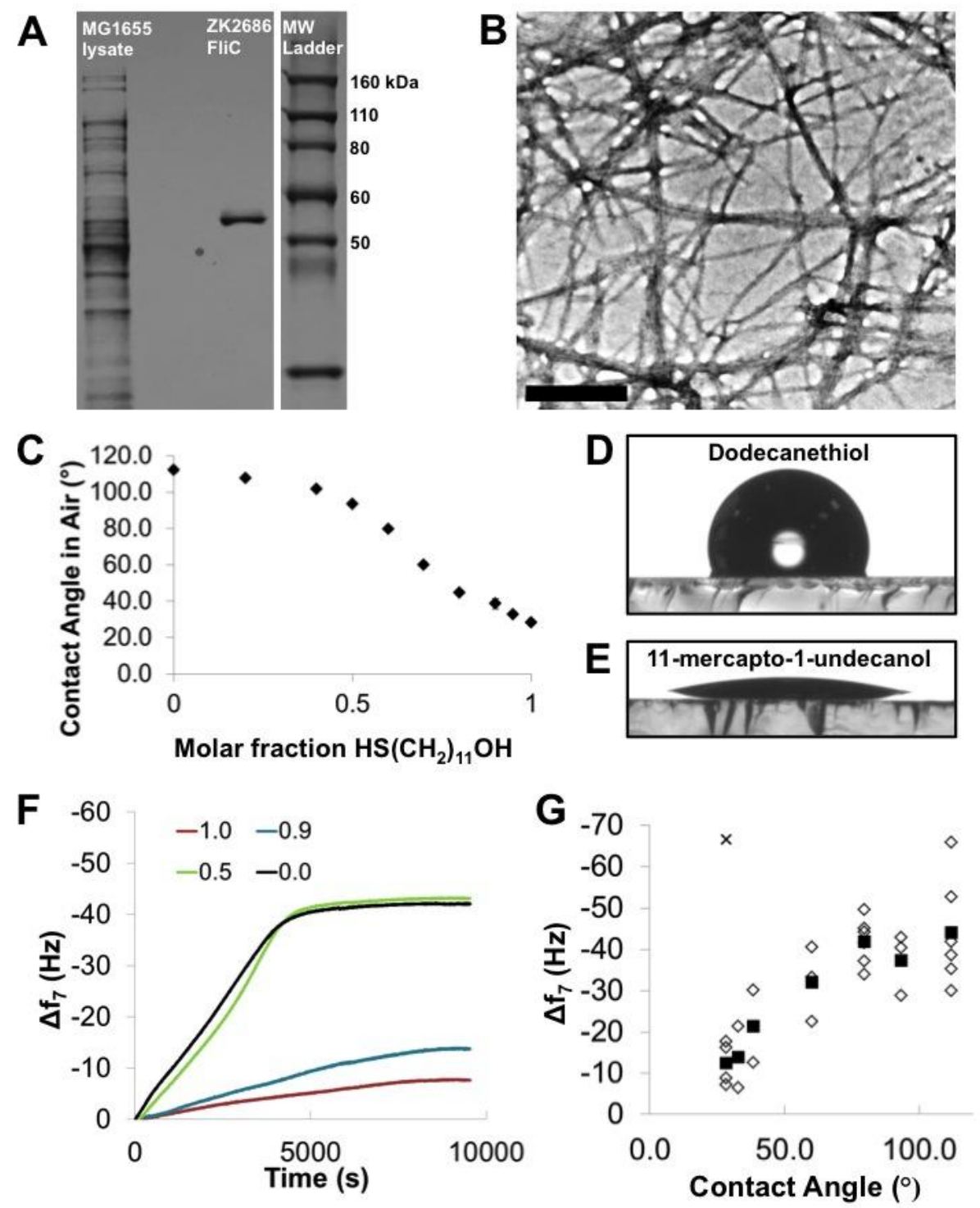

Figure 1. Increasing surface hydrophobicity improves adhesion of isolated flagella. (A) Sodium dodecyl sulfate polyacrylamide gel electrophoresis of MG1655 whole cell lysate (left, for comparison) and purified flagellin (FliC, middle), stained with Coomassie Blue. Molecular 
weights (MW) as indicated in MW ladder (right). (B) Flagella are purified in filamentous form. TEM image of purified flagellar filaments negatively stained with $1 \%$ phosphotungstic acid. Scale bar is $500 \mathrm{~nm}$. (C) Contact angle of a water droplet on mixed thiol SAM surfaces, in air; averaged over 5 measurements on two separate samples. SAMs are composed of a blend of 11mercapto-1-undecanol and dodecanethiol in the fractions indicated. Water droplets are shown on (D) the most hydrophobic (100\% dodecanethiol) SAM surface, and (E) the most hydrophilic (100\% 11-mercapto-1-undecanol) SAM surface. $(F)$ Frequency change of QCM resonance $\left(7^{\text {th }}\right.$ overtone) during flow of isolated flagella. Fraction 11-mercapto-1-undecanol for each SAM (as in $C$ ) is indicated for each trace in the legend. (G) Final frequency changes in $7^{\text {th }}$ overtone of QCM resonances for surfaces of varying hydrophobicity. Contact angle of each surface is plotted against the corresponding frequency change from several individual experiments per condition. Individual experiments are plotted as diamonds and means are plotted as squares. One outlier is included and indicated with an $\mathrm{x}$. 


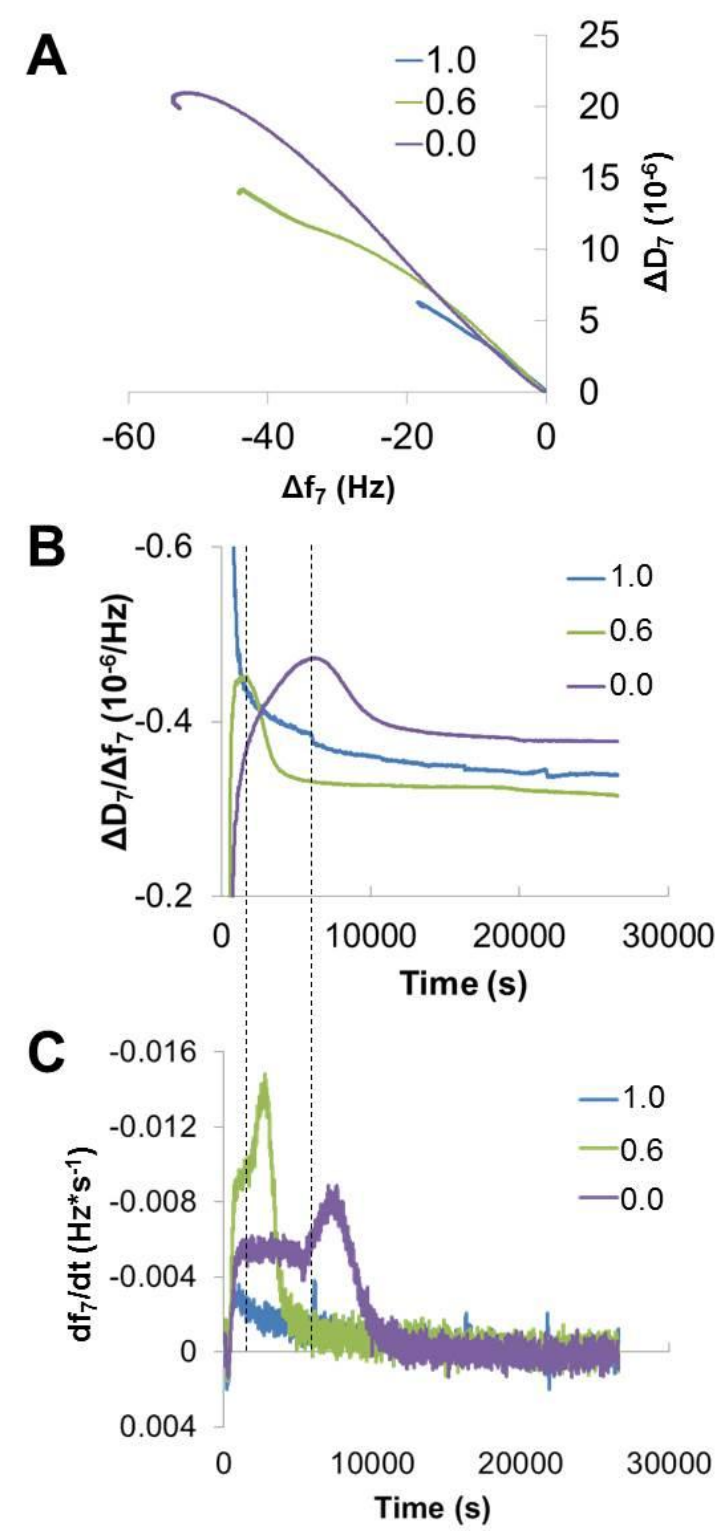

Figure 2. Energy dissipation and rate of adhesion of flagella. (A) Energy dissipation change plotted against frequency change for representative experiments. (B) Normalized energy dissipation from QCM-D. (C) Rate of change of QCM resonance frequency during flow of flagella over surface. Molar fraction (11-mercapto-1-undecanol/[11-mercapto-1-undecanol + dodecanethiol]) for each SAM is indicated for each trace in the legend. Traces of corresponding colors in all panels are from the same experiments. Dashed lines indicate times of peak normalized energy dissipation. 

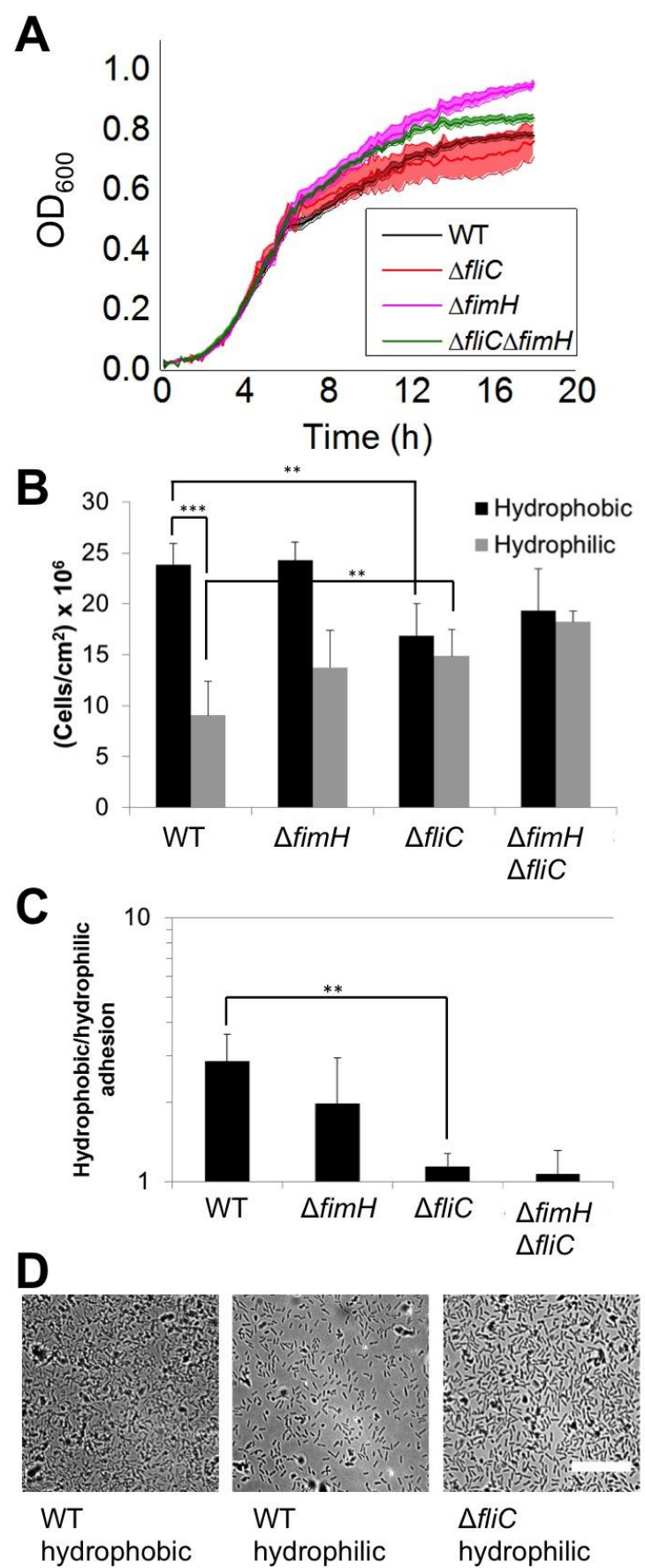

Figure 3. Flagella augment cell adhesion to hydrophobic surfaces, but reduce adhesion to hydrophilic surfaces. (A) Growth curves. Cells were inoculated in $\mathrm{M} 63^{+}$in a plate reader and $\mathrm{OD}_{600}$ was plotted over $18 \mathrm{~h}$ for the strains indicated. Shaded areas correspond to the standard deviation of 3-4 growth curves. (B) Number of adherent cells after $4 \mathrm{~h}$ of culture and rinsing of hydrophobic (dodecanethiol) and hydrophilic (11-mercapto-1-undecanol) SAM surfaces. Wildtype cells and mutants were characterized, as indicated. (C) Ratio of adherent cells per unit area 
on hydrophobic versus hydrophilic SAM surfaces for the genotypes indicated. Each data point is the ratio of adherent cells from a hydrophobic sample to the corresponding hydrophilic sample in the same well. Significance in $A$ and $B$ was assessed using Student's two-tailed t-test; ***, $p<0.001 ;{ }^{* *}, p<0.01$. (D) Transmitted light optical microscope images of adherent $E$. coli, with conditions as indicated. These are representative images from those used for quantification. Scale bar is $25 \mu \mathrm{m}$.

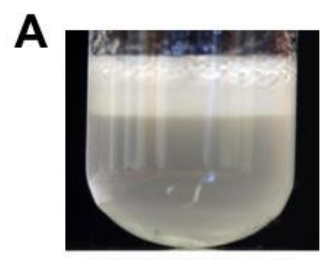

WT, 10 min rest

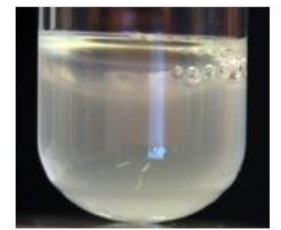

$\Delta$ flic, 10 min rest

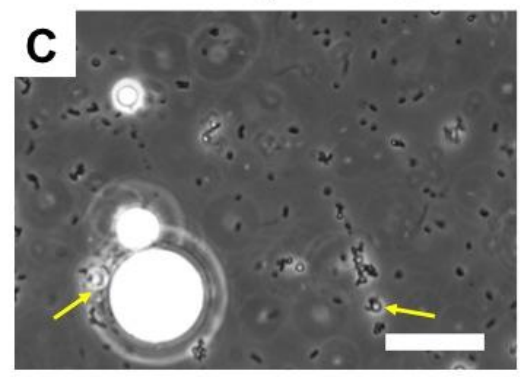

B

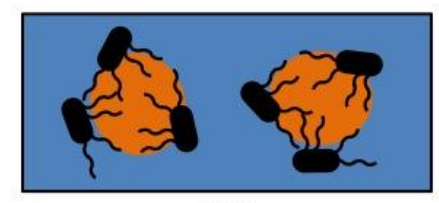

WT

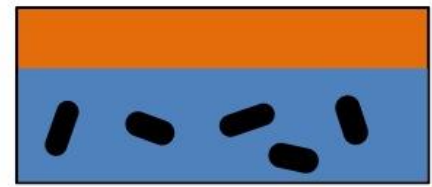

$\Delta$ flic

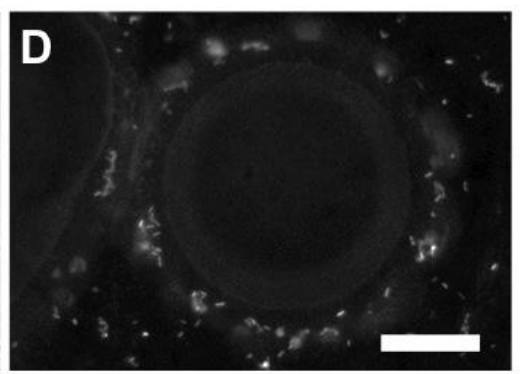

Figure 4. Bacterial emulsions demonstrate overall amphiphilicity of flagellated cells and hydrophilicity of $\Delta f l i C$ mutants. (A) Exponential phase aqueous bacterial cultures (lower phase) were mixed with dodecane (upper phase) and vortexed for $60 \mathrm{~s}$. After a 10 min rest, tubes were photographed. WT cells were photographed again after $2 \mathrm{~h}$, as indicated. (B) Schematic illustration of WT bacterial emulsions (upper panel) and phase separation of $\triangle \mathrm{fliC}$ cultures (lower panel). (C) Phase contrast optical micrograph of wild-type, exponential-phase E. coli from a dodecane-aqueous emulsion. The left arrow is pointing to an area of cell enrichment surrounding a large droplet of dodecane. The right-most arrow is pointing to a small droplet of dodecane stabilized by bacteria. (D) Fluorescence micrograph of Syto9-stained E. coli 
surrounding a large dodecane droplet. Note the enrichment of bacteria around the droplet and the lack of cells within the droplet. Scale bars in $C$ and $D$ are $25 \mu \mathrm{m}$.

\section{Tables:}

\begin{tabular}{|c|c|c|}
\hline Strain & Description & $\frac{\text { Reference or }}{\text { source }}$ \\
\hline ZK2686 & $\mathrm{W} 3110, \Delta(\operatorname{argF}-l a c) \mathrm{U} 169$ & 60 \\
\hline JW5665-1 & $\begin{array}{l}\mathrm{BW} 25113, \mathrm{~F}-, \Delta(\operatorname{ara\mathrm {D}}-\operatorname{ara\mathrm {B}}) 567, \\
\Delta l a c Z 4787(:: r r n \mathrm{~B}-3), \lambda-, \Delta b c s A 787:: \mathrm{kan}, \mathrm{rph}-1, \\
\Delta(r h a \mathrm{D}-r h a \mathrm{~B}) 568, h s d \mathrm{R} 514\end{array}$ & CGSC \\
\hline JW4277-1 & 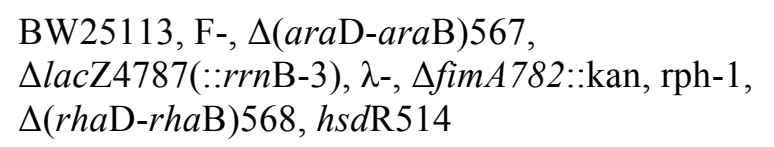 & $"$ \\
\hline JW1025-1 & $\begin{array}{l}\mathrm{BW} 25113, \mathrm{~F}-, \Delta(\operatorname{ara} \mathrm{D}-\operatorname{araB}) 567, \\
\Delta l a c Z 4787(:: r r n \mathrm{~B}-3), \lambda-, \Delta \operatorname{csg} A 784:: \mathrm{kan}, \mathrm{rph}-1, \\
\Delta(r h a \mathrm{D}-r h a \mathrm{~B}) 568, h s d \mathrm{R} 514\end{array}$ & $"$ \\
\hline JW 1881-1 & $\begin{array}{l}\text { BW25113, F-, } \Delta(\operatorname{araD}-\operatorname{araB}) 567, \\
\Delta l a c Z 4787(:: r r n \mathrm{~B}-3), \lambda-, \Delta f l h D 745:: \mathrm{kan}, \mathrm{rph}-1, \\
\Delta(r h a \mathrm{D}-r h a \mathrm{~B}) 568, h s d \mathrm{R} 514\end{array}$ & $"$ \\
\hline JW1908-1 & 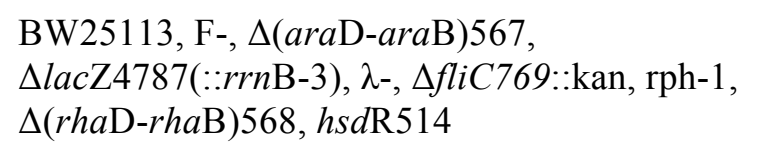 & $"$ \\
\hline JW 1878-4 & $\begin{array}{l}\text { BW25113, F-, } \Delta(\operatorname{araD}-\operatorname{araB}) 567, \\
\Delta l a c Z 4787(:: r r n \mathrm{~B}-3), \lambda-, \Delta m o t B 742:: \mathrm{kan}, \mathrm{rph}-1, \\
\Delta(r h a \mathrm{D}-r h a \mathrm{~B}) 568, h s d \mathrm{R} 514\end{array}$ & $"$ \\
\hline RSF31 & ZK2686, $\Delta b c s A 787:: \mathrm{kan}$ & This study \\
\hline RSF30 & ZK2686, $\Delta$ fimA782::kan & $"$ \\
\hline RSF28 & ZK2686, $\Delta \operatorname{csg} A 784:: \mathrm{kan}$ & $"$ \\
\hline RSF29 & ZK2686, $\Delta f l h D 745:: \mathrm{kan}$ & $"$ \\
\hline RSF43 & ZK2686, $\Delta f l i C 769:: \mathrm{kan}$ & $"$ \\
\hline RSF41 & ZK2686, $\Delta \operatorname{motB} 742:: \mathrm{kan}$ & $"$ \\
\hline
\end{tabular}

CGSC, Coli Genetic Stock Center (Yale); kan, kanamycin resistance gene; cam, chloramphenicol resistance gene.

Table 1. Names, descriptions and sources for all E. coli strains used in this study.

\section{Acknowledgments:}

We thank Tanya Shirman for TEM imaging, Hera Vlamakis for her help with phage transduction, Matthew Cabeen for his assistance with large-scale cultures for purification, and 
Karen Fahrner for assistance with flagella purification protocols. This work was partially funded by the Office of Naval Research under the award N00014-11-1-0641.

\section{References:}

(1) Hall-Stoodley, L.; Costerton, J.; Stoodley, P. Bacterial Biofilms: From the Natural Environment to Infectious Diseases. Nat. Rev. Microbiol. 2004, 2, 95-108.

(2) Costerton, J.; Cheng, K.; Geesey, G.; Ladd, T.; Nickel, J.; Dasgupta, M.; Marrie, T. Bacterial Biofilms in Nature and Disease. Annu. Rev. Microbiol. 1987, 41, 435-464.

(3) Costerton, J. W.; Stewart, P. S.; Greenberg, E. P. Bacterial Biofilms: A Common Cause of Persistent Infections. Science 1999, 284, 1318-1322.

(4) Characklis, W. Bioengineering Report: Fouling Biofilm Development: A Process Analysis. Biotechnol. Bioeng. 1981, 23, 1923-1960.

(5) Delauney, L.; Compère, C.; Lehaitre, M. Biofouling Protection for Marine Environmental Sensors. Ocean Sci. 2010, 6, 503-511.

(6) Manov, D. V. M.; Chang, G. C.; Dickey, T. D. Methods for Reducing Biofouling of Moored Optical Sensors. Journal of atmospheric and oceanic technology 2004, 21, 958968.

(7) Dunne, W. Bacterial Adhesion: Seen Any Good Biofilms Lately? Clin. Microbiol. Rev. 2002, 15, 155.

(8) Banerjee, I.; Pangule, R. C.; Kane, R. S. Antifouling Coatings: Recent Developments in the Design of Surfaces That Prevent Fouling by Proteins, Bacteria, and Marine Organisms. Adv. Mater. 2011, 23, 690-718.

(9) Tuson, H. H.; Weibel, D. B. Bacteria-surface Interactions. Soft Matter 2013, 9, 4368.

(10) Cassie, A.; Baxter, S. Wettability of Porous Surfaces. Trans. Faraday Soc. 1944, 40, 546551.

(11) Bhushan, B.; Jung, Y. Natural and Biomimetic Artificial Surfaces for Superhydrophobicity, Self-Cleaning, Low Adhesion, and Drag Reduction. Prog. Mater Sci. 2011, 56, 1-108.

(12) Quéré, D. Non-Sticking Drops. Rep. Prog. Phys. 2005, 68, 2495-2532.

(13) Liu, K.; Yao, X.; Jiang, L. Recent Developments in Bio-Inspired Special Wettability. Chem. Soc. Rev. 2010, 39, 3240-3255.

(14) Friedlander, R. S.; Vlamakis, H.; Kim, P.; Khan, M.; Kolter, R.; Aizenberg, J. Bacterial Flagella Explore Microscale Hummocks and Hollows to Increase Adhesion. Proc. Natl. Acad. Sci. U. S. A. 2013, 110, 5624-5629.

(15) Ong, Y.-L.; Razatos, A.; Georgiou, G.; Sharma, M. M. Adhesion Forces between E. Coli Bacteria and Biomaterial Surfaces. Langmuir 1999, 15, 2719-2725.

(16) Hermansson, M. The DLVO Theory in Microbial Adhesion. Colloids Surf. B Biointerfaces 1999, 14, 105-119.

(17) Poortinga, A. T.; Bos, R.; Norde, W.; Busscher, H. J. Electric Double Layer Interactions in Bacterial Adhesion to Surfaces. Surf. Sci. Rep. 2002, 47, 1-32.

(18) Busscher, H.J,; Uyen, M. H. W. J. C.; van Pelt, A. W. J.; Weerkamp, A. H.; Arends, J. Kinetics of adhesion of the oral bacterium S. sanguis $\mathrm{CH} 3$ to polymers with different surface free energies. Appl. Environ. Microbiol. 1986, 51, 910-914

(19) Busscher ., H.J.; Norde, W.; Sharma, P.K.; van der Mei, h.C.; Interfacial re-arrangement in initial microbial adhesion to surfaces. Current Opinion in Colloid and Interface Science 
2010, 15, 510-517

(20) Pratt, L.; Kolter, R. Genetic Analysis of Escherichia Coli Biofilm Formation: Roles of Flagella, Motility, Chemotaxis and Type I Pili. Mol. Microbiol. 1998, 30, 285-293.

(21) Bos, R.; Mei, H.; Busscher, H. Physico chemistry of Initial Microbial Adhesive Interactions-its Mechanisms and Methods for Study. FEMS Microbiol. Rev. 1999, 23, 179230.

(22) Ahrens, R.; Ott, M.; Ritter, A.; Hoschützky, H.; Bühler, T.; Lottspeich, F.; Boulnois, G. J.; Jann, K.; Hacker, J. Genetic Analysis of the Gene Cluster Encoding Nonfimbrial Adhesin I from an Escherichia Coli Uropathogen. Infect. Immun. 1993, 61, 2505-2512.

(23) Troge, A.; Scheppach, W.; Schroeder, B. O.; Rund, S. A.; Heuner, K.; Wehkamp, J.; Stange, E. F.; Oelschlaeger, T. A. More than a Marine Propeller - the Flagellum of the Probiotic Escherichia Coli Strain Nissle 1917 Is the Major Adhesin Mediating Binding to Human Mucus. Int. J. Med. Microbiol. 2012, 302, 304-314.

(24) Roy, K.; Hilliard, G.; Hamilton, D.; Luo, J.; Ostmann, M.; Fleckenstein, J. Enterotoxigenic Escherichia Coli EtpA Mediates Adhesion between Flagella and Host Cells. Nature 2009, 457, 594-598.

(25) Thomas, W. E.; Nilsson, L. M.; Forero, M.; Sokurenko, E. V.; Vogel, V. Shear-Dependent "stick-and-Roll" Adhesion of Type 1 Fimbriated Escherichia Coli. Mol. Microbiol. 2004, 53, 1545-1557.

(26) Müller, C. M.; Aberg, A.; Straseviçiene, J.; Emody, L.; Uhlin, B. E.; Balsalobre, C. Type 1 Fimbriae, a Colonization Factor of Uropathogenic Escherichia Coli, Are Controlled by the Metabolic Sensor CRP-cAMP. PLoS Pathog. 2009, 5, e1000303.

(27) Schwartz, D. J.; Kalas, V.; Pinkner, J. S.; Chen, S. L.; Spaulding, C. N.; Dodson, K. W.; Hultgren, S. J. Positively Selected FimH Residues Enhance Virulence during Urinary Tract Infection by Altering FimH Conformation. Proceedings of the National Academy of Sciences 2013.

(28) Pinkner, J. S.; Remaut, H.; Buelens, F.; Miller, E.; Åberg, V.; Pemberton, N.; Hedenström, M.; Larsson, A.; Seed, P.; Waksman, G.; et al. Rationally Designed Small Compounds Inhibit Pilus Biogenesis in Uropathogenic Bacteria. Proceedings of the National Academy of Sciences 2006, 103, 17897-17902.

(29) Gophna, U.; Barlev, M.; Seijffers, R.; Oelschlager, T. A.; Hacker, J.; Ron, E. Z. Curli Fibers Mediate Internalization of Escherichia Coli by Eukaryotic Cells. Infect. Immun. 2001, 69, 2659-2665.

(30) Norinder, B. S.; Lüthje, P.; Yadav, M.; Kadas, L.; Fang, H.; Nord, C. E.; Brauner, A. Cellulose and PapG Are Important for Escherichia Coli Causing Recurrent Urinary Tract Infection in Women. Infection 2011, 39, 571-574.

(31) Connell, I.; Agace, W.; Klemm, P.; Schembri, M.; Mărild, S.; Svanborg, C. Type 1 Fimbrial Expression Enhances Escherichia Coli Virulence for the Urinary Tract. Proc. Natl. Acad. Sci. U. S. A. 1996, 93, 9827-9832.

(32) Olsén, A.; Jonsson, A.; Normark, S. Fibronectin Binding Mediated by a Novel Class of Surface Organelles on Escherichia Coli. Nature 1989, 338, 652-655.

(33) Cegelski, L.; Pinkner, J. S.; Hammer, N. D.; Cusumano, C. K.; Hung, C. S.; Chorell, E.; Aberg, V.; Walker, J. N.; Seed, P. C.; Almqvist, F.; et al. Small-Molecule Inhibitors Target Escherichia Coli Amyloid Biogenesis and Biofilm Formation. Nat. Chem. Biol. 2009, 5, 913-919.

(34) Otto, K.; Elwing, H.; Hermansson, M. The Role of Type 1 Fimbriae in Adhesion of 
Escherichia Coli to Hydrophilic and Hydrophobic Surfaces. Colloids Surf. B Biointerfaces 1999, 15, 99-111.

(35) Walker, S. L.; Redman, J. A.; Elimelech, M. Role of Cell Surface Lipopolysaccharides in Escherichia Coli K12 Adhesion and Transport. Langmuir 2004, 20, 7736-7746.

(36) Genevaux, P.; Muller, S.; Bauda, P. A Rapid Screening Procedure to Identify Mini-Tn10 Insertion Mutants of Escherichia Coli K-12 with Altered Adhesion Properties. FEMS Microbiol. Lett. 1996, 142, 27-30.

(37) Wood, T.; González Barrios, A.; Herzberg, M.; Lee, J. Motility Influences Biofilm Architecture in Escherichia Coli. Appl. Microbiol. Biotechnol. 2006, 72, 361-367.

(38) Domka, J.; Lee, J.; Bansal, T.; Wood, T. K. Temporal Gene-Expression in Escherichia Coli K-12 Biofilms. Environ. Microbiol. 2007, 9, 332-346.

(39) Crawford, R.; Reeve, K.; Gunn, J. Flagellated but Not Hyperfimbriated Salmonella Enterica Serovar Typhimurium Attaches to and Forms Biofilms on Cholesterol-Coated Surfaces. $J$. Bacteriol. 2010, 192, 2981-2990.

(40) Erdem, A.; Avelino, F.; Xicohtencatl-Cortes, J.; Girón, J. Host Protein Binding and Adhesive Properties of H6 and H7 Flagella of Attaching and Effacing Escherichia Coli. $J$. Bacteriol. 2007, 189, 7426-7435.

(41) Thomason, L.; Costantino, N.; Court, D. E. Coli Genome Manipulation by P1 Transduction. Curr. Protoc. Mol. Biol. 2007, Chapter 1, Unit 1.17.

(42) Serra, D. O.; Richter, A. M.; Klauck, G.; Mika, F.; Hengge, R. Microanatomy at Cellular Resolution and Spatial Order of Physiological Differentiation in a Bacterial Biofilm. MBio 2013, 4, e00103-13.

(43) Sánchez, B.; López, P.; González-Rodríguez, I.; Suárez, A.; Margolles, A.; Urdaci, M. C. A Flagellin-Producing Lactococcus Strain: Interactions with Mucin and Enteropathogens. FEMS Microbiol. Lett. 2011, 318, 101-107.

(44) Arora, S.; Ritchings, B.; Almira, E.; Lory, S.; Ramphal, R. The Pseudomonas Aeruginosa Flagellar Cap Protein, FliD, Is Responsible for Mucin Adhesion. Infect. Immun. 1998, 66, 1000 .

(45) Tasteyre, A.; Barc, M.; Collignon, A.; Boureau, H.; Karjalainen, T. Role of FliC and FliD Flagellar Proteins of Clostridium Difficile in Adherence and Gut Colonization. Infect. Immun. 2001, 69, 7937.

(46) Zgair, A. K.; Chhibber, S. Adhesion of Stenotrophomonas Maltophilia to Mouse Tracheal Mucus Is Mediated through Flagella. J. Med. Microbiol. 2011, 60, 1032-1037.

(47) Hayashi, F.; Smith, K. D.; Ozinsky, A.; Hawn, T. R.; Yi, E. C.; Goodlett, D. R.; Eng, J. K.; Akira, S.; Underhill, D. M.; Aderem, A. The Innate Immune Response to Bacterial Flagellin Is Mediated by Toll-like Receptor 5. Nature 2001, 410, 1099-1103.

(48) Miyamoto, Y.; Iimura, M.; Kaper, J. B.; Torres, A. G.; Kagnoff, M. F. Role of Shiga Toxin versus H7 Flagellin in Enterohaemorrhagic Escherichia Coli Signalling of Human Colon Epithelium in Vivo. Cell. Microbiol. 2006, 8, 869-879.

(49) Bain, C. D.; Troughton, E. B.; Tao, Y. T.; Evall, J.; Whitesides, G. M.; Nuzzo, R. G. Formation of Monolayer Films by the Spontaneous Assembly of Organic Thiols from Solution onto Gold. J. Am. Chem. Soc. 1989, 111, 321-335.

(50) Love, J. C.; Estroff, L. A.; Kriebel, J. K.; Nuzzo, R. G.; Whitesides, G. M. Self-Assembled Monolayers of Thiolates on Metals as a Form of Nanotechnology. Chem. Rev. 2005, 105, 1103-1169.

(51) Bertilsson, L.; Liedberg, B. Infrared Study of Thiol Monolayer Assemblies on Gold: 
Preparation, Characterization, and Functionalization of Mixed Monolayers. Langmuir 1993, 9, 141-149.

(52) Laibinis, P. E.; Nuzzo, R. G.; Whitesides, G. M. Structure of Monolayers Formed by Coadsorption of Two N-Alkanethiols of Different Chain Lengths on Gold and Its Relation to Wetting. J. Phys. Chem. 1992, 96, 5097-5105.

(53) Arima, Y.; Iwata, H. Effect of Wettability and Surface Functional Groups on Protein Adsorption and Cell Adhesion Using Well-Defined Mixed Self-Assembled Monolayers. Biomaterials 2007, 28, 3074-3082.

(54) Höök, F.; Rodahl, M.; Brzezinski, P.; Kasemo, B. Energy Dissipation Kinetics for Protein and Antibody-Antigen Adsorption under Shear Oscillation on a Quartz Crystal Microbalance. Langmuir 1998.

(55) Owen, R. J.; Crocker, J. C.; Verma, R.; Yodh, A. G. Measurement of Long-Range Steric Repulsions between Microspheres due to an Adsorbed Polymer. Phys. Rev. E Stat. Nonlin. Soft Matter Phys. 2001, 64, 011401.

(56) Bevan, M. A.; Prieve, D. C. Forces and Hydrodynamic Interactions between Polystyrene Surfaces with Adsorbed PEO-PPO-PEO. Langmuir 2000, 16, 9274-9281.

(57) Boks, N. P.; Norde, W.; van der Mei, H. C.; Busscher, H. J. Forces Involved in Bacterial Adhesion to Hydrophilic and Hydrophobic Surfaces. Microbiology 2008, 154, 3122-3133.

(58) Howell, C.; Vu, T. L.; Lin, J. J.; Kolle, S.; Juthani, N.; Watson, E.; Weaver, J. C.; Alvarenga, J.; Aizenberg, J. Self-Replenishing Vascularized Fouling-Release Surfaces. ACS Appl. Mater. Interfaces 2014, 6, 13299-13307.

(59) Rosenberg, M.; Gutnick, D. Adherence of Bacteria to Hydrocarbons: A Simple Method for Measuring Cell-Surface Hydrophobicity. FEMS 1980.

(60) Danese, P.; Pratt, L.; Kolter, R. Exopolysaccharide Production Is Required for Development of Escherichia Coli K-12 Biofilm Architecture. J. Bacteriol. 2000, 182, 3593. 
TOC Graphic:
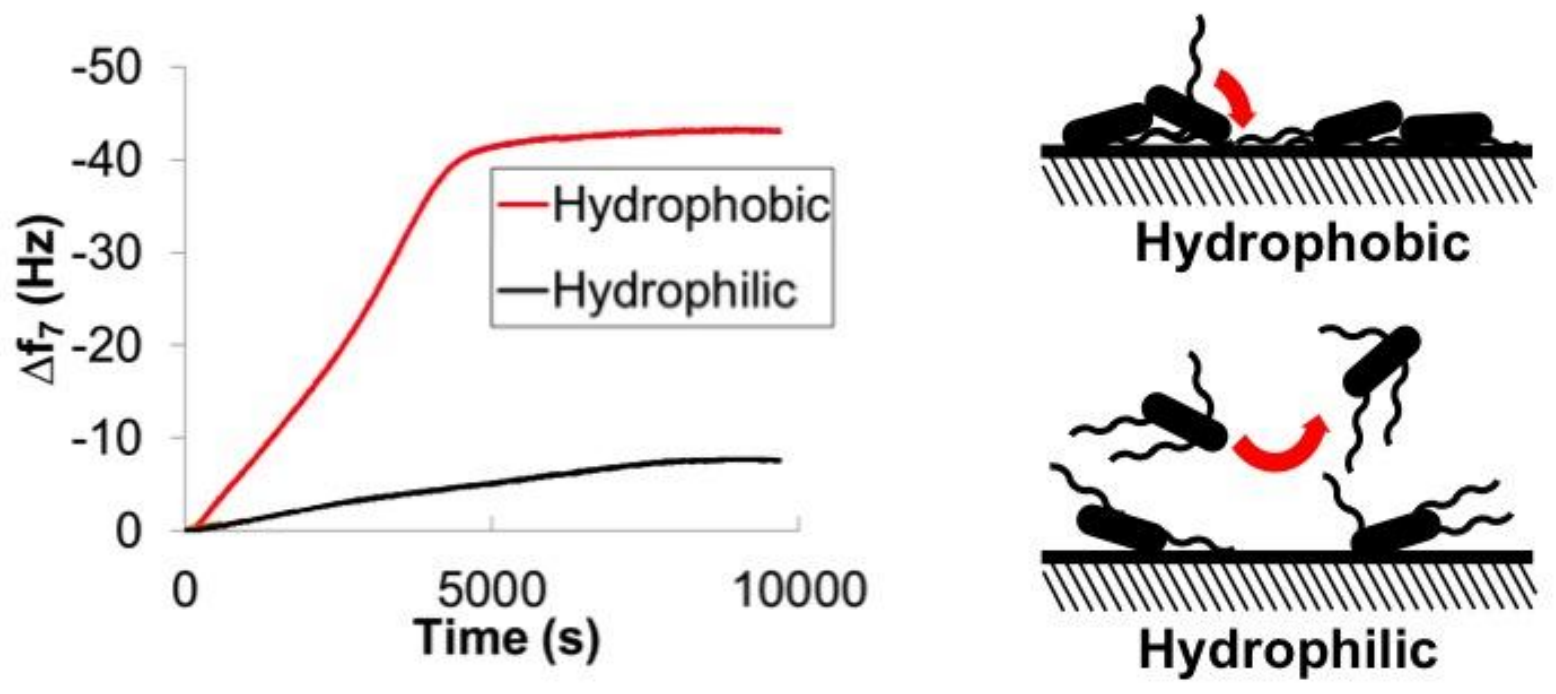